\title{
Experiences of Adults Using \\ Continuous Subcutaneous Insulin Infusion: A Qualitative Study
}

\author{
Naglaa Ibrahim Mesbah ${ }^{a} \quad$ Nehad Abd-El-Raheem Taha ${ }^{a}$ Zahra Nema Rahme ${ }^{a}$ \\ Faten Fawzi Sukkar ${ }^{\mathrm{a}}$ Dina Mohamed Omar ${ }^{\mathrm{b}}$ \\ ${ }^{a}$ Department of Education and Training, Dasman Diabetes Institute, Kuwait City, Kuwait; \\ ${ }^{b}$ Department of Clinical Services, Dasman Diabetes Institute, Kuwait City, Kuwait
}

\section{Highlights of the Study}

- This is a qualitative case study on adult users of continuous subcutaneous insulin infusion.

- Developing psychosocial evaluation prior to the initiation of insulin infusion would set expectations of therapy and help clinicians decide the eligibility of patients for insulin infusion.

- Regular education should be provided by nurse educators to maintain the psychosocial wellbeing of patients who suffer from fear of pump malfunction.

\section{Keywords}

Type 1 diabetes mellitus · Quality of life · Insulin pump therapy

\begin{abstract}
Objectives: We aimed to determine the quality of life (QoL) of Kuwaiti adults with type 1 diabetes mellitus (T1DM) who used continuous subcutaneous insulin infusions (CSIIs) and to understand their perception of this treatment. Background: CSII is an effective and safe alternative to multiple daily injections for T1DM, with the potential to improve both glycemic control and QoL. Although CSII has been widely used in Kuwait, its effectiveness and benefits have not been properly evaluated. Healthcare providers and patients need more information on CSII before its initiation. Methods: In this qualitative case study, semi-structured individual interviews were performed using a topic guide. Interviews were
\end{abstract}

stopped at data saturation point. Subsequently, a thematic analysis of transcripts was performed. Results: Eighteen adults with T1DM (8 males and 10 females; age $31.4 \pm 6.4$ years; diabetes duration $17.7 \pm 9.5$ years; CSII duration $3.6 \pm$ 2.4 years) were recruited from the CSII clinic. Six main themes were studied: health benefits, lifestyle flexibility, improvement of mood and emotion, practical problems, physical and personal effects, and self-confidence to manage diabetes. CSII positively affected the QoL of participants', most notably by enhancing lifestyle flexibility; however, important negative qualities were also reported. Conclusion: The identified themes highlight the complexity of the experiences of participants, and can be used to provide a framework to help healthcare providers understand how people with T1DM become aware of their treatment regimens. It is important to identify the positive aspects of CSII and not focus only on its negative effects.

(c) 2019 The Author(s)

Published by S. Karger AG, Basel

\begin{tabular}{ll}
\hline KARGER & $\begin{array}{l}\text { Ko 2019 The Author(s) } \\
\text { Published by S. Karger AG, Basel Operger }\end{array}$ \\
karger@karger.com & This is an Open Access article licensed under the Creative Commons \\
www.karger.com/mpp & $\begin{array}{l}\text { Attribution-NonCommercial-4.0 International License (CC BY-NC) } \\
\text { (http://www.karger.com/Services/OpenAccessLicense), applicable to } \\
\text { the online version of the article only. Usage and distribution for com- } \\
\text { mercial purposes requires written permission. }\end{array}$
\end{tabular}

Nehad Abdul-Raheem Taha

Department of Education and Training

Dasman Diabetes Institute

PO Box 1180, Dasman, Kuwait City 15462 (Kuwait)

E-Mail nehad.taha@dasmaninstitute.org 


\section{Introduction}

Continuous subcutaneous insulin infusions (CSIIs) is an option to facilitate intensive insulin therapy without increasing the risk of hypoglycemia [1]. Advantages of CSII include delivering doses more accurately, reducing blood glucose variability, reducing the risk of hypoglycemia and severe hypoglycemia, improving glycemic control, and eliminating the need for multiple daily injections (MDIs). CSII also allows flexibility in bolusing insulin, exercising, eating scheduling, varying carbohydrate intake, and eliminating the unpredictable effects of longacting insulin using different basal rates [2]. In fact, the hourly basal rate imitates the physiological requirements [3].

Not only can CSII delay or prevent complications of diabetes, but it can also improve the quality of life (QoL) [4]. QoL benefits for both the patient and family include the feeling of control, freedom, flexibility, and independence [5]. Negative effects on QoL include the persistent reminder of the disease, need to wear the pump continuously, device visibility, and fear of malfunction [6]. These may also interfere with sexual activity and sleep [7], and weight gain is more likely [6]. Finally, the cost can be an issue if the therapy is not covered by insurance providers. However, this is not relevant in Kuwait, where full coverage is provided by the Ministry of Health.

Diabetes management, including frequent self-monitoring and carbohydrate counting, is time-consuming and disruptive to daily life, often resulting in distress [8]. Maintaining psychological wellbeing should be the cornerstone of diabetic care because of its close relationship to physical wellbeing [9]. Understanding the experiences and perceptions of people with different management regimes is necessary to achieve that goal. Therapeutic and QoL benefits are associated with improved outcomes from the complex psychosocial issues in diabetes [9]. However, the psychosocial impact and experience of CSII use in adults have been largely ignored. Studies on QoL using various assessment tools have yielded contradictory findings [10].

In Kuwait, the incidence of type 1 diabetes mellitus (T1DM) has increased 20 -fold in the last 20 years [11] and, moreover, more adults are using CSII. Many studies have explored the experiences and views of injection users, but very few have focused on pump users [12]. Given that there appear to be no published studies related to this topic in Kuwait, we performed qualitative research to explore the impact of CSII on QoL, focusing on individuals' qualitative perceptions of CSII as a tool for dia-
Table 1. Participant demographics

\begin{tabular}{|c|c|c|c|c|c|c|}
\hline $\begin{array}{l}\text { Partici- } \\
\text { pant No. }\end{array}$ & $\begin{array}{l}\text { Age, } \\
\text { years }\end{array}$ & Gender & $\begin{array}{l}\text { Marital } \\
\text { status }\end{array}$ & $\begin{array}{l}\text { Duration } \\
\text { of T1DM, } \\
\text { years }\end{array}$ & $\begin{array}{l}\text { MDI ex- } \\
\text { perience, } \\
\text { years }\end{array}$ & $\begin{array}{l}\text { CSII ex- } \\
\text { perience, } \\
\text { years }\end{array}$ \\
\hline 1 & 21 & Female & Single & 14 & 12 & 2 \\
\hline 2 & 49 & Male & Married & 20 & 18.5 & 1.5 \\
\hline 3 & 38 & Female & Married & 30 & 28 & 2 \\
\hline 4 & 25 & Male & Single & 15 & 7 & 8 \\
\hline 5 & 33 & Female & Single & 9 & 5 & 4 \\
\hline 6 & 35 & Female & Married & 34 & 30 & 4 \\
\hline 7 & 36 & Male & Married & 26 & 23 & 3 \\
\hline 8 & 35 & Male & Married & 27 & 18 & 9 \\
\hline 9 & 28 & Female & Single & 19 & 15 & 4 \\
\hline 10 & 26 & Female & Single & 16 & 14 & 2 \\
\hline 11 & 28 & Female & Single & 15 & 12 & 3 \\
\hline 12 & 36 & Male & Married & 30 & 23 & 7 \\
\hline 13 & 31 & Female & Married & 23 & 18 & 5 \\
\hline 14 & 35 & Female & Single & 4 & 2 & 2 \\
\hline 15 & 27 & Male & Married & 2 & 1 & 1 \\
\hline 16 & 28 & Male & Married & 20 & 15 & 5 \\
\hline 17 & 27 & Female & Married & 13 & 11 & 2 \\
\hline 18 & 28 & Male & Married & 3 & 2 & 1 \\
\hline Mean & 31.4 & & & 17.7 & 14.1 & 3.6 \\
\hline SD & 6.4 & & & 9.5 & 8.6 & 2.3 \\
\hline
\end{tabular}

betes management. Ultimately, we hope this will inform clinical decision-making, patient selection, and education to improve QoL and glycemic control in patients with T1DM.

\section{Materials and Methods}

Design

Given the cultural context of the study, it was important to adopt an exploratory case study approach, in which individual experience of living with and managing diabetes using CSII is the main focus of the study.

\section{Participants}

The inclusion criteria were Kuwaiti males and females aged 21 years and older who were diagnosed with T1DM, using CSII for at least 12 months before the interview, and had received MDIs for at least 6 months before starting CSII. Non-Arabic speakers and those with learning disabilities and mental or physical illnesses were excluded. Purposive sampling was used for recruitment from our insulin pump clinic [13]. The sample size was not predetermined but rather defined by the data saturation point [14].

\section{Data Collection}

Eighteen subjects were individually interviewed using a semistructured interview topic guide with open-ended questions. Prompts were used to encourage the interviewees to express their experiences, thoughts, and feelings towards CSII. All interviews were recorded digitally. 
Table 2. Emergent themes

1. Health benefits

- Better glycemic control

- Reduction in hypoglycemia attacks

- Reduction in hypoglycemia severity

- Reduction in glucose variability

- Reduction in hospital admission

- Reduction in omitting insulin doses

4. Practical trouble fear

- Fear of pump malfunction

- Fear of kinked cannula

- Fear of air bubbles

- Fear with alarms
2. Lifestyle flexibility

- Flexibility of eating

- Flexibility of exercise

- Better quality of sleep

- Flexibility of social and work life

- Enabling fasting during Ramadan

- Flexibility during travelling

5. Physical and personal influence

- Less pain

- Fewer lumps and scars

- Risk of local reactions

- Pump visibility

- Less freedom in clothes choices
3. Mood and emotion improvement

- Less frequent injections

- Better glycemic control

- Better relationship with healthcare team

- Less worry about complications

- Less arguing with family members

- Less worry of forgetting insulin

6. Self-confidence

- Gaining knowledge

- Applying information

- Problem solving

- Less dependent

\section{Data Analysis}

All 18 interviews were transcribed, then analyzed parallel to the interviews to monitor for data saturation, which was considered to be reached when themes started to be repeated [14]. The recordings were transcribed verbatim, before being translated from Arabic to English. The translation was reviewed twice by 3 researchers to confirm that the original meaning was retained. Data analysis was performed using an inductive thematic approach. Data from transcripts were then categorized into codes or groups of expressions or phrases that were common across the transcripts. Similar codes were categorized into subthemes. Further analysis was performed to identify the main themes. Finally, the themes and codes were reviewed to verify the effectiveness of the transcript analysis and to reduce bias [15]. To ensure rigor, emergent codes and themes were reviewed and analyzed by the 4 authors separately. The findings reflect the consensus reached after discussion by the 4 researchers.

\section{Results}

Eighteen individuals were required to achieve data saturation ( 8 males and 10 females; mean age $31.4 \pm 6.4$ years; mean diabetes duration $17.7 \pm 9.5$ years; mean CSII duration $3.6 \pm 2.4$ years; Table 1). Interviews lasted $25-35 \mathrm{~min}$ on average for each participant. Most participants compared their old MDI regimen to the current CSII regimen. We identified six main themes, which are presented with relevant subthemes in Table 2 .

\section{Health Benefits}

All participants highlighted the benefits of getting alerts from the sensor-augmented pumps and explained how this feature helped them avoid high or low blood sugar compared with the MDI regimen, resulting in fewer hospital admissions and hypoglycemic episodes. Sixteen participants reported less blood glucose variability compared to the MDI regimen. Another benefit was that participants woke up less frequently at night to pass urine, reflecting better glycemic control with CSII than with MDI. Five participants intentionally omitted doses while eating snacks, dining outdoors, and even with main meals. Four participants omitted insulin injections with exercise to avoid hypoglycemia during or after exercise. All participants expressed social embarrassment associated with taking insulin injections in public or at social events, so they tended to omit insulin doses but reported that this issue was significantly improved with CSII use (Table 2, Textbox 1).

\section{Lifestyle Flexibility}

All participants reported an overall improvement in QoL, mostly in terms of lifestyle flexibility, such as eating times, food selection, exercise, travelling, social life, and work life. Among these, greater flexibility in eating was highlighted by all participants, and they reported that CSII helped them live a nearly normal life by allowing improved food selection, portion selection, and eating times. All participants reported that exercise safety was also improved by CSII device features, such as insulin suspend and temporary change to the basal rate. These options significantly improved their blood glucose control during and after exercise, allowing greater involvement in physical activities.

Participants reported many negative effects of MDI on their social lives, which were alleviated using CSII. They now feel less restrained during family visits. The 12 participants who were working stated that they had become more productive at work and required fewer sick leaves. Moreover, they feel more relieved as they no longer have to "hide" to take an insulin injection in case they need to eat. 
Ramadan, the holy month for Muslims, requires fasting from sunrise to sunset. Unfortunately, many people with T1DM are advised not to fast to avoid hypoglycemia or hyperglycemia. Ten participants reported that they could fast most days after switching to CSII. This could be achieved by using insulin suspend or temporary basal options. Regarding travelling, participants reported feeling generally more comfortable than with MDI. However, they found it annoying when airport security staff inquired about the pump (Table 2, Textbox 2).

\section{Mood and Emotional Improvement}

All participants were contented since using CSII, with the reduced frequency of injections and better glycemic control cited as common reasons. All participants described pumps as their artificial pancreas that improved their lives and reduced their fears of developing diabetic complications. Thirteen participants stated that CSII allowed them to lead more normal family lives, and they had fewer arguments with family members about meal portions, meal timing, blood glucose monitoring, and physical activities. Fewer arguments meant that they felt more relaxed and happy, which seemed to extend to other family members (Table 2, Textbox 3 ).

\section{Practical Problems}

Sixteen participants were fearful of being dependent on a machine, the malfunction of which could interrupt the insulin delivery to their bodies. All participants had experienced sporadic mechanical problems, such as kinked cannulas, local skin trauma during insertion, and air bubbles in the tube. However, they also received support from their diabetes team or the manufacturers of the pumps for troubleshooting (Table 2, Textbox 4).

\section{Physical and Personal Effects}

Ten female participants were concerned about the appearance of the pumps and the resulting limitations in clothing options. Specifically, they did not want to declare their diabetes and wanted to wear certain styles of clothing, such as party dresses and traditional Kuwaiti dresses (i.e., the abaya). Eight male participants did not view these as significant issues (Table 2, Textbox 5).

\section{Self-Confidence to Manage Diabetes}

All participants stated that their knowledge and skills about diabetes self-management and carbohydrate counting were much enhanced, positively reflecting on confidence in managing their diabetes. There were four subthemes: gaining knowledge, applying information, problem solving, and being less dependent (Table 2, Textbox 6).

\section{Discussion}

We identified six themes, namely health benefits, lifestyle flexibility, mood and emotion improvement, practical problems, physical and personal effects, and self-confidence to manage diabetes. Together, these themes reflected the complexity of the experiences of participants and the impact of CSII on different aspects of their lives.

The first theme showed that CSII positively enhanced glycemic control, affected health, as evidenced by reduced glucose variability, and fewer missed boluses. Indeed, several studies have objectively confirmed such an effect $[16,17]$.

The second theme concerned the significant impact of CSII on QoL. We noted that CSII improved flexibility in several aspects of life, including eating schedules, meal portions, exercise, sleep, social life, work, fasting for Ramadan, and travelling. It has been suggested that such lifestyle flexibility is the most significant factor affecting QoL [18]. Indeed, several researchers have reported that CSII use significantly improved QoL, citing the main benefits as increased flexibility, freedom, and self-dependence; these can improve social lives, eating, socialization, daily exercise, and sleep routines $[12,18,19]$. Other studies have also indicated substantial psychosocial benefits associated with CSII [20].

A previous study similarly reported that patients who intentionally omit insulin are aware of the potential risks [21]. However, these patients prefer to take those risks rather than suffer embarrassment, interference with daily activities, or injection-related pain [21]. Because most patients with diabetes prefer to have as few insulin injections per day as possible [21], they often do not take additional correction injections. This contrasts with CSII users who frequently use boluses for correction [19]. Participants of this study emphasized the simplicity of taking insulin via the pump, which is consistent with the evidence that CSII can improve patient adherence to treatment [22].

All study participants highlighted the value of flexibility in dose and meal timing. Unlike patients receiving MDI, it has been shown that CSII users require fewer extra snacks to avoid hypoglycemia and do not need fixed meal timing [23]. Additionally, CSII provides flexibility when travelling or needing a bolus dose, making it espe- 
cially useful for shift workers, workers in sensitive jobs, and business travelers. The ability to modify the basal rates also means less need for snacks during and after exercise [24]. In fact, CSII use may reduce post-exercise hyperglycemia and late-onset hypoglycemia [25].

When fasting during Ramadan, people with T1DM on MDI regimens are at high risk of hypoglycemia, hyperglycemia, DKA, and dehydration [26]. In the present study, participants were able to fast safely, which is consistent with previous research findings [27].

In agreement with other studies, our participants reported that they experienced social stigma with traditional injecting, and that this had a negative effect on disease management [10]. Participants typically felt embarrassed when injecting themselves in public, so they may inject at suboptimal sites or may delay injections when away from home, or even avoid social activities [10]. In contrast, our participants reported that CSII made it more convenient for them to receive insulin anywhere. It should be noted that McMahon et al. [28] reported no significant improvements in social life or QoL among patients who switched to CSII from MDI.

The third theme showed that CSII improved the overall mood of participants due to reduced injection frequency, better glycemic control, fewer arguments with family, and less worry about complications and missing insulin doses. Pickup and Harris [29] illustrated that mood can improve if diabetes-related social stressors with family, friends, and work are relieved. They also showed that better glycemic control reduced worry about developing diabetic complications. Concerning the relationship with HCPs, Todres et al. [18] highlighted that CSII required extensive education and follow-up that strengthened the relationship and improved patients' moods. Similar to our results, Wilson [30] found that mood improvement was simply due to the fewer number of injections and relieving the burden of carrying insulin injections [19].

The fourth theme concerned technical problems with pump use. Similar to the findings of Low et al. [19] our participants experienced sporadic mechanical problems with their pumps and were concerned about the interruption of insulin delivery, causing DKA [19]. Panic on alarms was common because it could indicate abnormal blood glucose levels or pump malfunction [19]. However, some participants simply perceived the alarms as a safety feature and useful reminder to take proper action (e.g., reminders to monitor blood glucose, change the pump battery, or check the insulin reservoir). Proper education on troubleshooting, which is a prerequisite for pump in- stallation, prepares users to deal with situations that require immediate action and help reduce such anxiety. Telecommunications with a diabetes educator or pump specialist also reduced the fear of technical malfunction. In worst-case scenarios, patients may disconnect the insulin pump and temporarily use insulin injections, emphasizing the importance of keeping back-up supplies of short- and long-acting insulin in case of emergencies.

Theme five related to the physical and personal effects of CSII use. We found that the opinions of our participants were divided. While some felt less pain, fewer lumps, and fewer scars, others reported greater discomfort due to pump visibility and restricted clothing choices. Overall, the physical effects of different treatments were a substantial concern. Pump visibility leads to feeling stigmatized [23]. Our participants reported being self-conscious and uncomfortable using such a device in public [19].

Notably, females were more concerned about their body image and social acceptance, expressing greater embarrassment with pump visibility, which is again consistent with previous research [23]. Moreover, women stated that the pump interfered with clothing choices $[12,19$, 23]. Some reported having to stitch a hidden pocket in abaya-style garments or having to clip the pump to their brassieres. Furthermore, they often felt forced to select looser-fitting clothes than usual and unable to find suitable clothes for parties. Therefore, they would disconnect the pump during such occasions. Males, in contrast, were comfortable wearing the pump in public and at parties. The sixth theme was about the enhanced self-confidence gained in managing diabetes with CSII. This was based on increased knowledge, problem-solving, and greater independence. Before starting CSII, patients underwent a structured training course that covered technical information, diabetes management, and problem-solving skills.

We conclude that improved lifestyle flexibility was the most notable theme. Reported benefits included better glycemic control, fewer or less severe hypoglycemia episodes, reduced glucose variability, fewer hospital admissions, and fewer omitted insulin doses. Lifestyle flexibility was reported to be enhanced in terms of eating, exercise, sleep quality, social and work life, travelling, and fasting during Ramadan. Additionally, mood was improved due to better glycemic control, a better relationship with healthcare professionals, less worry about complications, fewer family conflicts about diabetes, and less frequent injections. Fewer lumps and scars, as well as less pain, were the noted benefits of CSII. Concerns reported 
were fear of local reactions, reduced freedom in clothing choices, and pump visibility. Most CSII users experienced self-empowerment and self-confidence in managing diabetes; however, "being treated by a machine" was associated with practical problems, which was the main concern of the participants. With the increased use of CSII for management of T1DM, it is the responsibility of nurse educators to provide cognitive behavioral therapy to maintain psychosocial wellbeing for patients who suffer from fear pump malfunction. This study has highlighted the need to develop appropriate psychosocial evaluation prior to CSII initiation to help health professionals make more informed decisions about eligibility to CSII.

The success of CSII in terms of glycemic control has been investigated thoroughly. However, there is scant literature on the experiences and perceptions of adults using this therapy in the Arabian culture. It could be debated that the CSII is an expensive tool for T1D management; however,evidencehasshown that theimprovements in glycemic control associated with CSII leads to a reduction in the incidence and cost of diabetes-related complications [31]. These complications increase the cost of care and decrease the QoL. Even though QoL may improve marginally, the cost of managing diabetes-related complications is much higher in the long term than the cost of CSII. Thus, the cost of the CSII therapy is justifiable in the long run to allow governments to support covering the cost of CSII.

This study has confirmed many challenges, as well as facilitators associated with CSII therapy. Regular enforcement of education on dealing with pump malfunction and living with a pump is necessary and should be regularly provided. It is recommended that assessment of eligibility of patients for CSII should include negative aspects of its use. Although troubleshooting is already a part of pump training, CSII users should receive regular refresher sessions to prevent skill decay. Finally, patient expectations of CSII should be assessed to ensure parity between expectations and the deliverable results. Such insights may widen eligibility criteria to include more patients who could benefit from CSII. The identified themes represent a framework to help healthcare providers understand how people with T1DM perceive their treatment regimens, emphasizing the need to identify both the positive and negative effects of CSII.

The major limitation of this study is that it was conducted in a single center with a relatively small sample size. Therefore, this limits the ability to generalize the results and requires further studies from different settings.

\section{Acknowledgements}

We thank Dasman Diabetes Institute for offering opportunities for research and for offering the postgraduate program in diabetes care and education for healthcare professionals in Kuwait in collaboration with the University of Dundee. We are grateful to all the participants for their valuable time and inputs.

\section{Statement of Ethics}

Ethical approval was obtained from the Ethical Review Committee at Dasman Diabetes Institute prior to the study, and signed informed consent was provided by each participant. Data storage was secure, with access limited to researchers, but could be retrieved on request. All transcripts were coded and anonymized to maintain the confidentiality of the participants. Hard copies of transcripts were stored in a safe cabinet, and electronic copies were stored on an encrypted external storage device.

\section{Disclosure Statement}

The authors have no conflicts of interest to declare.

\section{Funding Sources}

The authors have no funding sources to disclose.

\section{Author Contributions}

N.I.M. is the principal investigator, performed, transcribed, and translated the interviews, and wrote the manuscript. N.A.T. and Z.N.R. observed the interviewing process. N.A.T., F.F.S., and D.M.O helped in writing and editing the manuscript and the tables. N.A.T. is the corresponding author. All authors confirmed the translation and the coding and reviewed and approved the manuscript.

\section{References}

1 Rubin RR, Peyrot M. Health-related quality of life and treatment satisfaction in the SensorAugmented Pump Therapy for A1C Reduction 3 (STAR 3) trial. Diabetes Technol Ther. 2012;14:143-51.

2 Boronat M, Sánchez-Hernández RM, Rodríguez-Cordero J, Jiménez-Ortega A, Nóvoa FJ. Suspension of basal insulin to avoid hypoglycemia in type 1 diabetes treated with insulin pump. Endocrinol Diabetes Metab Case Rep. 2015;2015:140081.

3 Danne T, von Schütz W, Lange K, Nestoris C, Datz N, Kordonouri O. Current practice of insulin pump therapy in children and adolescents - the Hannover recipe. Pediatr Diabetes. 2006 Aug;7(s4 Suppl 4):25-31. 
4 White D, Waugh N, Elliott J, Lawton J, Barnard K, Campbell MJ, et al.; REPOSE group. The Relative Effectiveness of Pumps over MDI and Structured Education (REPOSE): study protocol for a cluster randomised controlled trial. BMJ Open. 2014 Sep;4(9): e006204.

5 Barnard K. CSII: psychological aspects [internet]. 2014 Aug 13; Diapedia. Available from: h t t p s://doi org/10.14496/ dia.8104974843.6.014.

6 Lenhard MJ, Reeves GD. Continuous subcutaneous insulin infusion: a comprehensive review of insulin pump therapy. Arch Intern Med. 2001 Oct;161(19):2293-300.

7 Bruttomesso D, Pianta A, Crazzolara D, Scaldaferri E, Lora L, Guarneri G, et al. Continuous subcutaneous insulin infusion (CSII) in the Veneto region: efficacy, acceptability and quality of life. Diabet Med. 2002;19:62834.

8 Everett J, Kerr D. Measuring quality of life in diabetes. J Diabetes Nurs. 2001;5:53-5.

9 Barnard K, Skinner T. Cross-sectional study into quality of life issues surrounding insulin pump use in type 1 diabetes. Pract Diabetes Int. 2008;25:194-200.

10 Barnard KD, Lloyd CE, Skinner TC. Systematic literature review: quality of life associated with insulin pump use in Type 1 diabetes. Diabet Med. 2007;24:607-17.

11 Shaltout AA, Wake D, Thanaraj TA, Omar DM, Al-Abdul Razzaq D, Channanath A, et al. Incidence of type 1 diabetes has doubled in Kuwaiti children $0-14$ years over the last 20 years. Pediatr Diabetes. 2017;18(8):761-6.

12 Alsaleh FM, Smith FJ, Taylor KM. Experiences of children/young people and their parents, using insulin pump therapy for the management of type 1 diabetes: qualitative review. J Clin Pharm Ther. 2012:37:140-7.

13 Etikan I, Musa SA, Alkassim RS. Comparison of convenience sampling and purposive sampling. AJTAS. 2016:5(1):10.11648/j.ajtas.20160501.11.
14 Saunders B, Sim J, Kingstone T, Baker S, Waterfield J, Bartlam B, et al. Saturation in qualitative research: exploring its conceptualization and operationalization. Qual Quant. 2018;52(4):1893-907.

15 Turner DW 3rd. Qualitative interview design A practical guide for novice investigators. Qual Rep. 2010;15(3):754-60. Available from: http://www.nova.edu/ssss/QR/QR15-3/qid. pdf.

16 Battelino $\mathrm{T}$, Conget I, Olsen B, SchutzFuhrmann I, Hommel E, Hoogma R, et al. The use and efficacy of continuous glucose monitoring in type 1 diabetes treated with insulin pump therapy: a randomised controlled trial. Diabetologia. 2012;55:3155-62.

17 Bergenstal RM, Klonoff DC, Garg SK, Bode BW, Meredith M, Slover RH, et al. Thresholdbased insulin-pump interruption for reduction of hypoglycemia. N Engl J Med. 2013; 369:224-32.

18 Todres L, Keen S, Kerr D. Continuous subcutaneous insulin infusion in Type 1 diabetes: patient experiences of 'living with a machine'. Diabet Med. 2010 Oct;27(10):1201-4.

19 Low KG, Massa L, Lehman D, Olshan JS. Insulin pump use in young adolescents with type 1 diabetes: a descriptive study. Pediatr Diabetes. 2005;6:22-31.

20 Müller-Godeffroy E, Treichel S, Wagner VM, et al. Investigation of quality of life and family burden issues during insulin pump therapy in children with type 1 diabetes mellitus - a large-scale multicentre pilot study. Diabetic Med. 2009;26:493-501.

21 Peyrot M, Rubin RR, Kruger DF, Travis LB. Correlates of insulin injection omission. Diabet Care. 2010;33:240-5

22 Valenzuela JM, Patino AM, McCullough J, Ring C, Sanchez J, Eidson M, et al. Insulin pump therapy and health-related quality of life in children and adolescents with type 1 diabetes. J Pediatr Psychol. 2006;31:650-60.
23 Ritholz MD, Smaldone A, Lee J, Castillo A, Wolpert H, Weinger K. Perceptions of psychosocial factors and the insulin pump. Diabet Care. 2007;30:549-54.

24 Hanaire H, Lassmann-Vague V, Jeandidier N, Renard E, Tubiana-Rufi N, Vambergue A, et al. Treatment of diabetes mellitus using an external insulin pump: the state of the art. Diabet Metab. 2008;34:401-23.

25 Yardley JE, Iscoe KE, Sigal RJ, Kenny GP, Perkins BA, Riddell MC. Insulin pump therapy is associated with less post-exercise hyperglycemia than multiple daily injections: an observational study of physically active type 1 diabetes patients. Diabetes Technol Ther. 2013; 15:84-8.

26 Al-Arouj M, Assaad-Khalil S, Buse J, Fahdil I, Fahmy M, Hafez S, et al. Recommendations for management of diabetes during Ramadan update 2010. Diabet Care. 2010;33:1895-902.

27 Khalil AB, Beshyah SA, Abu Awad SM, Benbarka MM, Haddad M, Al-Hassan D, et al. Ramadan fasting in diabetes patients on insulin pump therapy augmented by continuous glucose monitoring: an observational real-life study. Diabetes Technol Ther. 2012;14:813-8.

28 McMahon SK, Airey FL, Marangou DA, McElwee KJ, Carne CL, Clarey AJ, et al. Insulin pump therapy in children and adolescents: improvements in key parameters of diabetes management including quality of life. Diabet Med. 2005;22:92-69.

29 Pickup JC, Harris A. Assessing quality of life for new diabetes treatments and technologies: a simple patient-centered score. J Diabetes Sci Technol. 2007;1:394-9.

30 Wilson V. Experiences of parents of young people with diabetes using insulin pump therapy. Paediatr Nurs. 2008;20:14-8.

31 Roze S, Valentine W, Zakrzewska K, Palmer A. Health-economic comparison of continuous subcutaneous insulin infusion with multiple daily injection for the treatment of type 1 diabetes in the UK. Diabet Med. 2005;22: 1239-45. 